\title{
MicroRNAs in Brain Function and Disease
}

\author{
Andreas Walter Kuss, $P h D$, and Wei Chen, $P h D$
}

\begin{abstract}
Corresponding author
Andreas Walter Kuss, PhD

Department for Human Molecular Genetics, Max Planck Institute for Molecular Genetics, Ihnestr. 73, D-14195, Berlin, Germany. E-mail: kuss_a@molgen.mpg.de
\end{abstract}

Current Neurology and Neuroscience Reports 2008, 8:190-197

Current Medicine Group LLC ISSN 1523-3804

Copyright (C) 2008 by Current Medicine Group LLC

MicroRNAs (miRNAs), a class of small, non-protein-coding transcripts about 21 nucleotides long, have recently entered center stage in the study of posttranscriptional gene regulation. They are now thought to be involved in the control of about one third of all protein-coding genes and play a role in the majority of cellular processes that have been studied. We focus on the role of the miRNA pathway in brain development, function, and disease by highlighting recent observations with respect to miRNAmediated gene regulation in neuronal differentiation, synaptic plasticity, and the circadian clock. We also discuss the implications of these findings with respect to the involvement of miRNAs in the etiopathology of brain disorders and pinpoint the emerging therapeutic potential of miRNAs for the treatment of human diseases.

\section{Introduction}

MicroRNAs (miRNAs) are small noncoding RNAs that regulate gene expression at the posttranscriptional level through sequence-specific base pairing with a target mRNA. Originally discovered in the nematode Caenorhabditis elegans in 1993 [1], lin-4, the first identified miRNA, was deemed an oddity peculiar to the worm until 7 years later, when the second miRNA found in C. elegans (let7) was shown to be conserved in both sequence and the expression pattern during development in a variety of organisms $[2,3]$. Intrigued by the idea that lin- 4 and let -7 might indeed be the first members of an abundant class of small regulatory RNAs to be discovered, several research groups started to clone small RNAs from diverse species and, consequently, hundreds of miRNAs were identified in worm, fly, and mammalian genomes [4-6]. Since then, numerous miRNAs have been detected in all multicellular eukaryotes that have been studied, as well as in a few viruses. Recently, the discovery of miRNAs in the unicellular algae Chlamydomonas reinhardtii indicated miRNA pathways as ancient regulatory mechanisms that evolved even before the emergence of multicellularity [7,8]. To date, in miRBase (http://microrna.sanger.ac.uk), the most carefully curated database of miRNAs with support from experimental evidence, over 5000 miRNA loci have been deposited, of which 541 are human (release 10.1). With the rapid development of next-generation sequencing technology and its application in small RNA discovery, the ongoing identification of new miRNAs is expected to accelerate even more in the near future $[9,10]$.

\section{miRNA Biogenesis}

A scheme representing the topical concept of miRNA biogenesis is shown in Figure 1. miRNAs are initially transcribed by RNA-polymerase II as long transcripts, often several kilobases in length, and termed pri-miRNAs. They are subsequently processed by the RNase III enzyme Drosha into approximately 70 -nucleotide hairpin RNAs [11]. Some spliced-out introns in Caenorhabditis elegans, Drosophila melanogaster, and mammals correspond exactly to pre-miRNAs and therefore do not

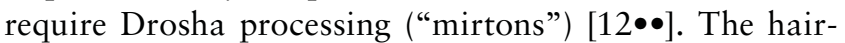
pin precursor miRNAs, called pre-miRNAs, are then exported from the nucleus to the cytoplasm [13] by the nuclear export factor exportin-5 in a guanosine-triphosphate-dependent manner. In the cytoplasm, they are then cleaved by Dicer, another RNase III enzyme, into imperfect double-stranded RNA duplexes with a 3' overhang of two nucleotides [14]. After unwinding of such an RNA duplex, the strand with lower thermodynamic stability at the $5^{\prime}$ end (also referred to as guide strand) is incorporated into a ribonucleoprotein (RNP) complex, called microRNP (miRNP), or miRNA-induced silencing complex (miRISC), a multicomponent protein complex responsible for silencing the target mRNA. The key components of miRISC are proteins of the argonaute (AGO) family. In mammals, four AGO proteins (AGO-1 to AGO-4) are involved in miRNA-mediated repression. In addition to AGOs, further regulatory proteins mediating miRISC function can be part of the complex. An example is the fragile $\mathrm{X}$ mental retardation protein (FMRP), an RNA- 


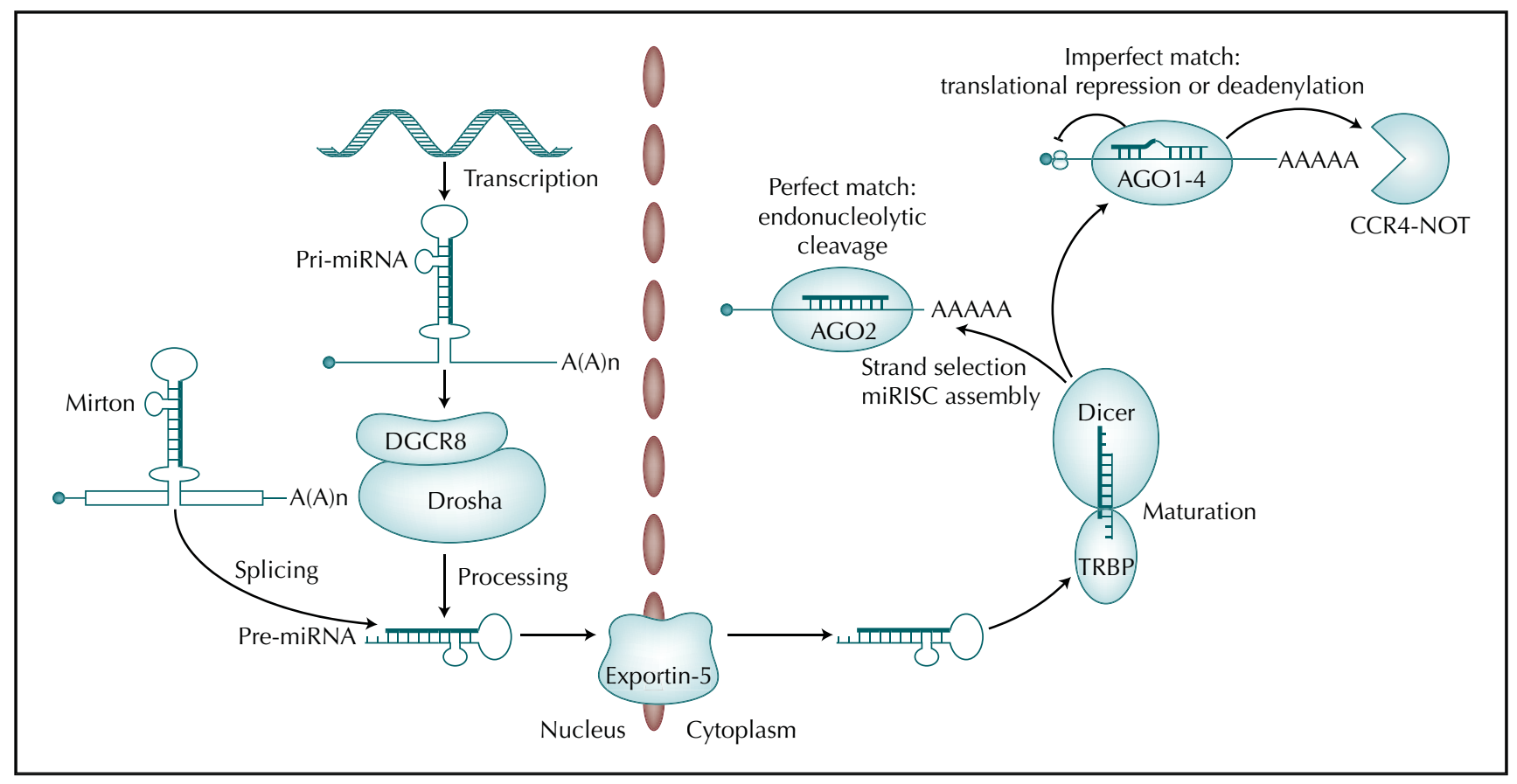

Figure 1. MicroRNAs (miRNAs) are encoded in the genome. Their genes are generally transcribed by RNA polymerase II and the transcripts can be spliced and polyadenylated. The primary transcript is referred to as pri-miRNA. The pri-miRNA is processed within the nucleus by an enzymatic complex containing the Drosha RNaselll enzyme and the DiGeorge syndrome critical region gene 8 (DGCR8) protein. The resulting precursor miRNA (pre-miRNA) is then exported to the cytoplasm by exportin-5. In the cytoplasm, the pre-miRNa is cleaved by Dicer in combination with the TAR RNA binding protein (TRBP). This yields an approximately 20-bp miRNA duplex, one strand of which is then selected to function as a mature miRNA, whereas the other strand is in most cases degraded. Mature miRNAs become part of a ribonucleoprotein (RNP) complex, called micro-RNP (miRNP), or an miRNA-induced silencing complex (miRISC). The miRISC recognizes its target and binds in the $3^{\prime}$ untranslated region of the target mRNA. When perfect complementarity exists between miRNA and target sequence, the target RNA is degraded. Without a perfect match, the target is not cleaved but is deadenylated or its translation is repressed. AGO-argonaute.

binding protein that acts as modulator of translation, particularly in neurons.

Depending on the degree of complementarity between the guide strand and its recognition site in the $3^{\prime}$ untranslated region (UTR) of the target, mRNA translation of the latter is repressed. A perfect match causes mRNA degradation via endonucleolytic cleavage, whereas an imperfect match leads to a suppression of protein expression due to both reduced translation efficiency and increased mRNA turnover. In animals, most miRNAs form imperfect base pairing with their binding sites [15]. At present, the molecular mechanism of such repression is still under debate. Evidence exists for various hypotheses, including inhibition of eukaryotic translation initiation factor 4E-dependent translation initiation, inhibition of translation elongation, premature translation termination, as well as mRNA decapping, deadenylation, and degradation [16-18]. Recently, miRNA regulation has gained a new dimension beyond its role as a translation repressor through the observation that, although they suppress translation in proliferating cells, miRNAs can also activate translation upon cell cycle arrest $[19 \bullet \bullet]$. Therefore, it is obvious that there is still much to be learned about these small molecules and that we are still at an early stage of understanding their importance for cellular differentiation and homeostasis.

\section{miRNA Targets}

Elucidation of miRNA function requires the identification of target genes, which remains a great challenge for most miRNAs identified in animals. It has, however, been shown that the interaction between miRNAs and their target mRNA in animals is primarily mediated through six to eight nucleotides in the $5^{\prime}$ part of miRNAs, known as miRNA seeds [20,21]. A diverse array of bioinformatic approaches has taken advantage of these seed sequences to predict miRNA targets. Based on bioinformatic prediction, it has been estimated that approximately one third of the protein-coding genes in the human genome are under the control of miRNA regulation [22]. Although the experimental verification of the targets has advanced much more slowly, an ever-increasing number of miRNAs have already been shown to function in a variety of biological processes, including developmental timing, apoptosis, differentiation, myogenesis, and glucose homeostasis [23]. In the following sections, we focus on the role of miRNAs in brain function and disease.

\section{miRNA and Brain Function}

Since the discovery of the C. elegans lin- 4 gene $[1,2]$, our understanding of the role of miRNAs in brain develop- 
ment and function has been steadily growing. Recent years have seen a tremendous increase in the number of miRNAs recognized as being either specifically expressed or at least enriched in the mammalian nervous system [24,25].

Evidence for an involvement of miRNAs has been found in many stages of the developing nervous system, including neural patterning, neuronal specification, and axonal path finding. miRNAs also seem to play a role in mature neurons (eg, in synaptic plasticity).

\section{miRNA function in brain development and neuron/glia differentiation}

For investigating potentially neurodevelopmental functions of miRNAs, researchers have mostly relied on two strategies: 1) investigation of single miRNAs or 2) interference with miRNA biogenesis and thereby disruption of the entire cellular miRNA complement. Both approaches require caution, however-the former because miRNAs often belong to subfamilies in which functional redundancy cannot be excluded, and the latter because it can usually not be determined to what extent additional cellular functions are disrupted and in what way this disruption contributes to the observed phenotype. For example, mutations in the Drosophila Argonaute 1 protein (dAGO-1), a miRISC component that is essential for miRNA function, lead to a drastic reduction in neurons and glial cells [26]. However, although a dysfunctional miRNA system is a very plausible explanation, an impact on other functions of dAGO-1 cannot be entirely ruled out. Despite such limitations, however, converging results of a growing number of studies provide increasingly strong evidence for a central involvement of neuronal miRNAs in the development of the central nervous system (CNS).

As mentioned previously, one important aspect that seems to be influenced by miRNAs is neural patterning (ie, the patterning of the embryonic CNS along its anterior-posterior, dorsal-ventral, and left-right axes). The mechanisms behind neural patterning might involve miRNAs on various levels. Development of the anteriorposterior axis of the CNS, for example, is regulated by homeobox (Hox) genes, which are in turn targeted by miRNAs [27,28]. Studies in zebrafish deficient in the Dicer protein (and therefore devoid of functional miRNAs) revealed organizational defects during the formation of the embryonic neural plate and during its transformation into the neural tube in the knockout animals [29••].

Beyond their potential role in basic structural organization of the CNS, evidence is growing that miRNAs function in neuronal differentiation. The most detailed investigation of miRNA influence on cell fate and identity has been carried out on the main chemosensory neurons for chemotactic reactions to water-soluble attractants (ASE neurons) in C. elegans. These receptor neurons have the ability to spatialize inputs based on the expression of distinct chemoreceptor collectives defined during development. This difference in cell identity is based on the interaction of two miRNAs (lsy-6 and miR-273) and their transcription factor targets in a double-negative feedback loop [30-32]. In mice, the miRNAs miR-9 and miR-124a have been implicated in the decision of neural precursors between differentiation into neurons and assumption of a glial phenotype [33]. Interestingly, even in nonneuronal cells, expression of miR-124a converts the overall gene-expression pattern to a neuronal one $[34 \bullet \bullet, 35]$. miR-124a decreases the levels of hundreds of nonneuronal transcripts, so that its introduction into HeLa cells promotes a neuronal-like mRNA profile, whereas the transcriptional repressor, RE1 silencing transcription factor, has a reciprocal activity, inhibiting the expression of neuronal genes, including miR-124a, in nonneuronal cells [36•]. A similar role for miR-133b in dopamine neuron differentiation was recently reported. The authors first observed that mice lacking Dicer in specific dopamine neurons are born alive but later in life show a progressive loss of neurons in combination with clinical features resembling Parkinson's disease. Therefore, Dicer seems essential for neuronal survival, suggesting that the loss of miRNAs may be involved in the development and/or progression of Parkinson's disease $[37 \bullet, 38]$. In view of the fact that the transfer of cellular-derived small RNAs (including miRNAs) partially preserved the dopaminergic phenotype in cell culture [37•], it is likely that the absence of miRNAs, and not the lack of other potential Dicer-related functions, is involved in the neurodegenerative process. Further investigations implicated a negative-feedback regulatory loop between miR-133b and the transcription factor Pitx 3 in the regulation of dopaminergic neuron differentiation [37•].

Beyond proper cell differentiation, axonal path finding is mandatory for the establishment of functional circuitry in the nervous system during neural development. The first indications for an involvement of miRNAs were discovered in zebrafish, where abnormalities in axon guidance were observed in association with Dicer defects [29••]. These findings were corroborated by a study in rat cell cultures showing that developing axons contain functional miRISCs [39].

Another important aspect of CNS development is the massive loss of glia and neurons, which can range from $20 \%$ to $80 \%$ of differentiated cells, depending on the brain region. Degeneration of these cells by programmed cell death (apoptosis) accompanies neurogenesis and the expansion of brain size caused by the enlargement of neuronal cell bodies, myelination, expansion of dendritic trees, secretion of the extracellular matrix, and blood vessel arborization in the growing brain. Apoptotic processes are strongly influenced by miRNAs, and even though the bulk of the experimental evidence in this respect has been obtained by cancer research [40], it leaves little doubt that miRNAs also play a role in the developmental sequences that shape the mammalian brain.

\section{miRNAs in synaptic plasticity}

miRNAs are expressed in the fully differentiated neurons of the mature brain (in many cases at comparatively 
high levels), and a central feature that might be subject to miRNA regulation is the control of plasticity. Synaptic plasticity has two distinct phases: an early phase that is independent of protein synthesis and a late phase that is long term and requires protein synthesis [41]. Many investigations have argued strongly for the role of local translation within dendrites in the establishment of longterm plasticity [42-45]. Given the involvement of miRNAs in translation regulation, long-term plasticity is conceivably where miRNA-mediated modulation has its greatest effect on brain function. In a very recent study focusing on the separate quantification of microRNAs in the neuritic and somatic compartments, a small number of miRNAs were found to be relatively enriched in the dendrite [46]. A model for miRNA-mediated effects at the synapse has been proposed [47], suggesting an influence of miRNAs on transcripts whose translation is dependent, for example, on stimulation of the ionotropic glutamate receptors by glutamate. However, all models of miRNA involvement in synaptic plasticity to date remain speculative and are shrouded by the limited knowledge of mRNA routing from the nucleus to distinct cytoplasmic destinations.

\section{miRNAs and the circadian clock}

In mammals, the master circadian clock resides in the suprachiasmatic nuclei (SCN) of the hypothalamus and keeps a roughly 24-hour cycle that is similar in the physiologic processes of nearly all organisms. The circadian rhythm is under the endogenous control of clock genes but can respond to external cues (primarily daylight). Numerous complex human behaviors, such as understanding speech or performing music, depend on the ability of the brain to determine time, and evidence for an involvement of clock genes in mental diseases is mounting [48].

At the molecular level, the SCN clock has been modeled as interlocking transcriptional feedback loops that drive rhythmic expression of critical clock components. Cheng et al. $[49,50]$ recently discovered that two miRNAs might modulate the intrinsic pacemaker activity and resetting capacity of the SCN. The authors first found that the transcription of miR-132 and miR-219 was under the control of the cyclic adenosine monophosphate (cAMP) response element-binding protein (CREB), the transcription factor mediating gene expression that is elicited by light stimulation in the SCN. In addition, miR219 was also identified as the target of another critical element of the transcriptional network governing intrinsic circadian rhythm, the transcription factor CLOCK. The transcriptional regulation of these two miRNAs by CREB and CLOCK was then substantiated by their rhythmic expression within SCN and the light induction of miR132 expression. To explore the physiologic role of the two miRNAs in setting the circadian clock, they were knocked out in the mouse SCN and it was shown that miR-219 can regulate the length of the circadian day, whereas mir-R32 acts as a feedback inhibitor of light-induced clock reset- ting [49]. In the search for potential mechanisms by which miR-219 and miR-132 modulate the circadian clock, Cheng and Obrietan [50] identified 28 and 42 potential targets respectively, by applying a bioinformatics approach. Two of these, the regulatory factor X4 (RFX4) for miR-132 and the suprachiasmatic nucleus oscillatory protein (SCOP) for miR-219, were experimentally confirmed [49]. The observation that several putative miR-132 and miR-219 targets encode ion channels, together with the recent work implicating cellular excitability in the generation of circadian rhythm, prompted the authors to speculate about the roles of miR-132 and miR-219 in cellular excitability, which could indeed be corroborated by overexpression experiments in cultured cortical neurons [49]. Finally, the link between the two miRNAs and core clock timing processes was established by the demonstration of their ability to augment the CLOCK-dependent, BMA1-dependent, and depolarization-dependent expression of mPer1 in cultured cells as well as in transgenic mice. Taken together, the available evidence strongly suggests a central involvement of the miRNA pathway in the control of circadian rhythm. Still, future studies will have to substantiate these findings and provide answers to the questions of if and how miRNAs contribute to diseaserelated aspects of the circadian clock.

\section{miRNA and Human Diseases}

The vast majority of data associating miRNAs with human diseases came from cancer-related studies. In the past few years, a number of miRNAs have been implicated in the regulation of cancer-relevant pathways, such as proliferation and apoptosis, and several miRNAs have been identified that act as either tumor suppressors or oncogenes. The role of miRNAs in tumorigenesis, including miRNA deregulation in brain tumors, has been extensively reviewed $[51 \bullet, 52]$. We focus on the contribution of the miRNA pathway to the etiopathology of brain disorders.

The first neurologic disorder linked with the miRNA pathway was fragile $\mathrm{X}$ syndrome, the most common form of inherited mental retardation, with an estimated prevalence of 1 in 4000 male individuals and 1 in 8000 female individuals. Fragile X syndrome clinically manifests with moderate to severe mental retardation, macroorchidism, and distinct facial features and is caused by loss of FMR 1 gene function. In most cases, the causative mutation is the unstable expansion of a CGG repeat in the $5^{\prime}$ UTR of $F M R 1$, which results in hypermethylation of the FMR1 promoter and thus in transcriptional silencing [53,54]. The product of FMR1 is FMRP, an RNA-binding protein associated with polyribosomes as part of an mRNA RNP (mRNP). FMRP can act as a negative regulator of translation within and at the base of dendritic spines. The first evidence of a link between FMRP and the miRNA pathway came from the studies of protein components of Drosophila FMRP (dFMRP). These components con- 
tained a complex that identified dFMRP as a component of miRISC and revealed specific interactions between dFMRP and two functional miRISC proteins, dAGO-2 and Dicer. Subsequent work in mammalian cells showed that in addition to Dicer and dAGO-2, dFMRP was also associated with endogenous miRNA [55]. Therefore, it has been proposed that FMRP regulates a specific set of mRNAs via the miRNA pathway. This hypothesis was further supported by a study that demonstrated the genetic interactions between dFMRP and dAGO-1 [56]. In that study, increased apoptosis caused by overexpression of dFMRP was largely suppressed by heterozygous loss of dAGO-1, whereas the synaptic overgrowth at the neuromuscular junction due to the absence of dFMRP was exacerbated by heterozygous loss of dAGO-1. This modulation of dFMRP-mediated translational regulation by an miRISC component directly implicates the potential role of the miRNA pathway in fragile $\mathrm{X}$ syndrome.

FMRP and AGO were the first proteins to be established as translational repressors. However, in recent work by Vasudevan et al. [19••], AGO-2 and fragile X mental retardation-related protein1 (FXR1) were shown to act as translational activators via the miRNA pathway under cell cycle arrest. In this context, it will be interesting to see whether FMRP can form similar complexes with a positive-effect mRNA translation, and if so, whether this also contributes to the etiology of fragile $\mathrm{X}$ syndrome.

Given the involvement of the miRNA pathway in fragile $\mathrm{X}$ syndrome, it is intriguing to investigate the potential role of miRNA dysregulation in the disease pathogenesis of mental retardation in general. For this purpose, we screened 13 known brain-expressed X-chromosomal miRNAs in a cohort of 464 patients with nonsyndromic $\mathrm{X}$-linked mental retardation and found four nucleotide changes in three different miRNA genes [57]. Of the four changes, which are all located outside the mature miRNA sequences, one change found in miR-222 is close to the Drosha processing site and might influence miRNA processing under certain conditions. Interestingly, miR-222 is also part of a $1.27-\mathrm{Mb}$ deletion that is likely to be responsible for mental retardation [58].

Although changes in miRNA genes can affect processing efficiency or alter target specificity, genetic variation of miRNA target sequences in the $3^{\prime}$ UTR could also influence the interaction between miRNAs and their targets. The first example of rare variants identified in miRNA target sequences came from the study of Tourette's syndrome, where an identical variant in the 3' UTR of the candidate gene SLIT and Trk-like (SLITRK1) was found in two unrelated patients but was absent in 3600 control chromosomes. This rare variant was located in a predicted binding site for mir-189, an miRNA expressed in the brain region implicated in Tourette's syndrome. More importantly, a luciferase reporter assay suggested that the predicted site was indeed functional and that the variant strengthened the interaction between SLITRK1 and mir-189 [59•].
Parkinson's disease (PD) is a common neurodegenerative disorder that is caused by a gradual loss of midbrain dopaminergic neurons (DNs). As mentioned previously, the observation that mice with a conditional deletion of Dicer in postmitotic midbrain DNs exhibited a PD-like phenotype prompted Kim et al. [37•] to identify the differentially expressed miRNAs between the midbrain of PD patients and that of controls. miR-133b turned out to be specifically enriched in the midbrain from normal individuals but to be deficient in samples from PD patients. This result, however, contradicts their finding that miR$133 \mathrm{~b}$ suppressed the full differentiation of DNs in cell culture and suggests an additional role of miR-133b in DN function and the pathology of PD, which still needs further elaboration [37•]. Recently, miRNA dysregulation was studied in another neurodegenerative disorder, Huntington's disease (HD) [60]. HD is caused by a CAG expansion in the gene encoding the huntington protein, and patients with HD show progressive loss of cortical and striatal neurons associated with choreic movement and dementia. It was found that huntington interacts with REST and that mutant huntington inhibits the interaction, leading to a deregulation of the REST target [61]. Starting with the identification of miRNA targets of REST, Johnson et al. [60] measured the expression of these potential targets in the cortex of the mouse model of HD. Compared with that in control mice, the expression of four miRNAs (miR-28a, miR-124a, miR-132, and miR-135b) was significantly reduced in HD mice, and in particular the reduced expression of miR-124a was reflected by an upregulation of its mRNA targets. In a subsequent study in human samples, only miR-132 was found to be downregulated in HD patients, which led to the increased expression of its mRNA target p250GAP [60]. The authors claimed that miRNA dysregulation accompanies HD and suggested a model in which enhanced levels of nuclear REST in HD neurons lead to changes in the neuronal transcriptome both directly, via repression of target gene expression, and indirectly, via regulation of neuronal miRNA expression [60].

Schizophrenia is a common brain disorder that affects approximately $1 \%$ percent of the general population [62]. To search for the candidate miRNAs involved in the etiopathology of this disease, Perkins et al. [63] compared the expression of miRNAs from postmortem prefrontal cortex of individuals with schizophrenia with that of unaffected individuals. By using microarrays spotted with probes against 265 human miRNAs (at a false discovery rate of $5 \%$ ), the authors found 16 miRNAs to be differentially expressed: 15 showed decreased expression and one showed higher expression in the schizophrenia patients as compared with controls. For miRNAs residing in introns of protein-coding genes, they observed that the ratios of microarray expression levels of miRNA versus mRNA (of the respective host gene) were significantly different for four of the six hosted miRNAs distinguished by schizo- 
phrenia [63]. Therefore the authors hypothesized that the downregulation of miRNAs they observed is due to altered miRNA biogenesis rather than altered transcription of primiRNA. This is in keeping with the speculation that the potential involvement of the DiGeorge critical region gene 8 encoded protein (an RNA-binding protein that assists Drosha in the processing of miRNAs) in schizophrenia is via its regulation of miRNA biogenesis [63].

\section{Therapeutic Potential of the miRNA System}

Given the emerging evidence that miRNAs regulate pathways that are involved in various disease etiologies, researchers are now starting to look at the potential of miRNA-based therapeutics. At present, there are two strategies for the development of miRNA-based therapeutics.

The first is to suppress endogenous miRNAs. This can be achieved by using chemically modified antisense oligonucleotides complementary to mature miRNAs, such as 2'-O-methyl oligoribonucleotides (2-O-MeRNA), which have been successfully used for inhibiting miRNA function in mammalian cell cultures. Similar results have been obtained in a number of mouse tissues by intravenous or direct injection of antagomir, a cholesterol-conjugated 2-O-Me-RNA with two or three phosphorothioate modifications on each end [64,65]. An alternative approach based on the same strategy uses decoy mRNAs, which are also known as miRNA sponges. These are transcripts expressed from strong promoters, bearing multiple tandem binding sites of a given miRNA of interest. Inclusion of a central mismatch at the AGO-2 cleavage site prevents degradation of the miRNA sponge and enables it to stably associate with the target miRNA [66]. In cell cultures, the authors showed that their miRNA sponge was at least as effective as chemically modified antisense oligonucleotides.

The second miRNA-based therapeutic strategy involves either restoring expression of miRNAs, which is reduced in disease-associated tissues, or augmenting the naturally occurring miRNA in targeting aberrant transcripts for silencing. Because miRNAs are chemically identical to small interfering RNAs (siRNAs), this strategy will face the same challenges currently encountered by siRNA-based therapeutics, such as the problem of efficient systematic delivery to different tissues or off-target effects [67]. However, all the methods developed in the siRNA field can also easily be used to solve problems in the therapeutic application of miRNAs. The rapid pace in this area of research raises the hope for successful employment of small RNAs in the treatment of human diseases in the future.

\section{Conclusions}

The study of miRNAs has revealed that they form a family that is much larger than originally assumed and that they contribute to the regulation of a large proportion of protein-coding transcripts. In order to understand the complexity of this regulatory network, future research will have to go beyond single candidate validation and ultimately will require genomic and proteomic studies. The available evidence for a central role of the miRNA pathway in neuronal development and function is, however, already compelling, and the first steps toward elucidating the involvement of miRNAs in the etiology of human cognitive disorders have been made. The coming years will, in all likelihood, see a continuation of the rapid development in miRNA research, and one is tempted to hope that RNA interference-based therapies are not too far beyond the horizon.

\section{Acknowledgments}

We thank Dr. Lars Riff Jensen and Dr. Andreas Tzschach for helpful discussions during the preparation of the manuscript.

\section{Disclosures}

No potential conflicts of interest relevant to this article were reported.

\section{References and Recommended Reading}

Papers of particular interest, published recently, have been highlighted as:

- Of importance

- Of major importance

1. Lee RC, Feinbaum RL, Ambros V: The C. elegans heterochronic gene lin-4 encodes small RNAs with antisense complementarity to lin-14. Cell 1993, 75:843-854.

2. Reinhart BJ, Slack FJ, Basson M, et al.: The 21-nucleotide let-7 RNA regulates developmental timing in Caenorhabditis elegans. Nature 2000, 403:901-906.

3. Pasquinelli AE, Reinhart BJ, Slack F, et al.: Conservation of the sequence and temporal expression of let-7 heterochronic regulatory RNA. Nature 2000, 408:86-89.

4. Lee RC, Ambros V: An extensive class of small RNAs in Caenorhabditis elegans. Science 2001, 294:862-864.

5. Lagos-Quintana M, Rauhut R, Lendeckel W, Tuschl T: Identification of novel genes coding for small expressed RNAs. Science 2001, 294:853-858.

6. Lau NC, Lim LP, Weinstein EG, Bartel DP: An abundant class of tiny RNAs with probable regulatory roles in Caenorhabditis elegans. Science 2001, 294:858-862.

7. Molnar A, Schwach F, Studholme DJ, et al.: miRNAs control gene expression in the single-cell alga Chlamydomonas reinhardtii. Nature 2007, 447:1126-1129.

8. Zhao T, Li G, Mi S, et al.: A complex system of small RNAs in the unicellular green alga Chlamydomonas reinhardtii. Genes Dev 2007, 21:1190-1203.

9. Ruby JG, Jan C, Player C, et al.: Large-scale sequencing reveals 21U-RNAs and additional microRNAs and endogenous siRNAs in C. elegans. Cell 2006, 127:1193-1207.

10. Berezikov E, Thuemmler F, van Laake LW, et al.: Diversity of microRNAs in human and chimpanzee brain. Nat Genet 2006, 38:1375-1377.

11. Zeng Y, Cullen BR: Recognition and cleavage of primary microRNA transcripts. Methods Mol Biol 2006, 342:49-56. 
12.• Ruby JG, Jan CH, Bartel DP: Intronic microRNA precursors that bypass Drosha processing. Nature 2007, 448:83-86.

This is a report of an alternative pathway for miRNA biogenesis in which debranched introns mimic the structural features of pri-miRNAs to enter the miRNA-processing pathway without Drosha-mediated cleavage.

13. Lund E, Guttinger S, Calado A, et al.: Nuclear export of microRNA precursors. Science 2004, 303:95-98.

14. Ketting RF, Fischer SE, Bernstein E, et al.: Dicer functions in RNA interference and in synthesis of small RNA involved in developmental timing in C. elegans. Genes Dev 2001, 15:2654-2659.

15. Bartel DP: MicroRNAs: genomics, biogenesis, mechanism, and function. Cell 2004, 116:281-297.

16. Jackson RJ, Standart N: How do microRNAs regulate gene expression? Sci STKE 2007, 2007:re1.

17. Nilsen TW: Mechanisms of microRNA-mediated gene regulation in animal cells. Trends Genet 2007, 23:243-249.

18. Eulalio A, Rehwinkel J, Stricker M, et al.: Target-specific requirements for enhancers of decapping in miRNA-mediated gene silencing. Genes Dev 2007, 21:2558-2570.

19.•• Vasudevan S, Tong Y, Steitz JA: Switching from repression to activation: microRNAs can up-regulate translation. Science 2007, 318:1931-1934.

This article reports that two well-studied microRNAs (let-7 and the synthetic microRNA miRcxcr4) induce translation upregulation of target mRNAs upon cell cycle arrest, whereas they repress translation in proliferating cells.

20. Doench JG, Sharp PA: Specificity of microRNA target selection in translational repression. Genes Dev 2004, 18:504-511.

21. Brennecke J, Stark A, Russell RB, Cohen SM: Principles of microRNA-target recognition. PLoS Biol 2005, 3:e85.

22. Lewis BP, Burge CB, Bartel DP: Conserved seed pairing, often flanked by adenosines, indicates that thousands of human genes are microRNA targets. Cell 2005, 120:15-20.

23. Kloosterman WP, Plasterk RH: The diverse functions of microRNAs in animal development and disease. Dev Cell 2006, 11:441-450.

24. Cao X, Yeo G, Muotri AR, et al.: Noncoding RNAs in the mammalian central nervous system. Annu Rev Neurosci 2006, 29:77-103.

25. Krichevsky AM: MicroRNA profiling: from dark matter to white matter, or identifying new players in neurobiology. ScientificWorldJournal 2007, 7:155-166.

26. Kataoka Y, Takeichi M, Uemura T: Developmental roles and molecular characterization of a Drosophila homologue of Arabidopsis Argonaute1, the founder of a novel gene superfamily. Genes Cells 2001, 6:313-325.

27. Chopra VS, Mishra RK: "Mir”acles in hox gene regulation. Bioessays 2006, 28:445-448.

28. Iimura T, Pourquie O: Hox genes in time and space during vertebrate body formation. Dev Growth Differ 2007 , 49:265-275.

29.•• Giraldez AJ, Cinalli RM, Glasner ME, et al.: MicroRNAs regulate brain morphogenesis in zebrafish. Science 2005, 308:833-838.

This article presents compelling evidence showing that miRNAs can induce pronounced target mRNA degradation, initiated by removal of the poly(A) tail.

30. Chang S, Johnston RJ Jr, Frokjaer-Jensen C, et al.: MicroRNAs act sequentially and asymmetrically to control chemosensory laterality in the nematode. Nature 2004, 430:785-789.

31. Johnston RJ Jr, Chang S, Etchberger JF, et al.: MicroRNAs acting in a double-negative feedback loop to control a neuronal cell fate decision. Proc Natl Acad Sci U S A 2005, 102:12449-12454.

32. Johnston RJ, Hobert O: A microRNA controlling left/right neuronal asymmetry in Caenorhabditis elegans. Nature 2003, 426:845-849.
33. Krichevsky AM, Sonntag KC, Isacson O, Kosik KS: Specific microRNAs modulate embryonic stem cell-derived neurogenesis. Stem Cells 2006, 24:857-864.

$34 . \bullet \quad$ Lim LP, Lau NC, Garrett-Engele P, et al.: Microarray analysis shows that some microRNAs downregulate large numbers of target mRNAs. Nature 2005, 433:769-773.

This important study was the first to show a general effect of miRNAs on mRNA stability and it suggested the global control of cell identity by miRNAs.

35. Makeyev EV, Zhang J, Carrasco MA, Maniatis T: The MicroRNA miR-124 promotes neuronal differentiation by triggering brain-specific alternative pre-mRNA splicing. Mol Cell 2007, 27:435-448.

36. Conaco C, Otto S, Han JJ, and Mandel G: Reciprocal actions of REST and a microRNA promote neuronal identity. Proc Natl Acad Sci U S A 2006, 103:2422-2427.

This article discusses the likelihood of distinct spatiotemporal expression patterns that reflect specific roles in the coordination of gene expression profiles that characterize neuronal cell types.

37. Kim J, Inoue K, Ishii J, et al.: A MicroRNA feedback circuit in midbrain dopamine neurons. Science 2007, 317:1220-1224.

The authors report a negative-feedback loop between miR-133b and the transcription factor Pitx3, which regulates dopaminergic neuron differentiation, suggesting orchestrated action of miRNAs and transcription factors. They link miRNA function to PD.

38. Schaefer A, O'Carroll D, Tan CL, et al.: Cerebellar neurodegeneration in the absence of microRNAs. J Exp Med 2007, 204:1553-1558.

39. Hengst U, Cox LJ, Macosko EZ, Jaffrey SR: Functional and selective RNA interference in developing axons and growth cones. J Neurosci 2006, 26:5727-5732.

40. Slack FJ, Weidhaas JB: MicroRNAs as a potential magic bullet in cancer. Future Oncol 2006, 2:73-82.

41. Sutton MA, Schuman EM: Dendritic protein synthesis, synaptic plasticity, and memory. Cell 2006, 127:49-58.

42. Kang H, Schuman EM: A requirement for local protein synthesis in neurotrophin-induced hippocampal synaptic plasticity. Science 1996, 273:1402-1406.

43. Huber KM, Kayser MS, Bear MF: Role for rapid dendritic protein synthesis in hippocampal mGluR-dependent longterm depression. Science 2000, 288:1254-1257.

44. Huber KM, Roder JC, Bear MF: Chemical induction of mGluR5- and protein synthesis-dependent long-term depression in hippocampal area CA1. J Neurophysiol 2001, 86:321-325.

45. Aakalu G, Smith WB, Nguyen N, et al.: Dynamic visualization of local protein synthesis in hippocampal neurons. Neuron 2001, 30:489-502.

46. Kye MJ, Liu T, Levy SF, et al.: Somatodendritic microRNAs identified by laser capture and multiplex RT-PCR. RNA 2007, 13:1224-1234.

47. Kosik KS: The neuronal microRNA system. Nat Rev Neurosci 2006, 7:911-920.

48. Lamont EW, Legault-Coutu D, Cermakian N, Boivin DB: The role of circadian clock genes in mental disorders. Dialogues Clin Neurosci 2007, 9:333-342.

49. Cheng HY, Papp JW, Varlamova O, et al.: microRNA modulation of circadian-clock period and entrainment. Neuron 2007, 54:813-829.

50. Cheng HY, Obrietan K: Revealing a role of microRNAs in the regulation of the biological clock. Cell Cycle 2007, 6:3034-3035.

51. Esquela-Kerscher A, Slack FJ: Oncomirs - microRNAs with a role in cancer. Nat Rev Cancer 2006, 6:259-269.

This is a recommended review on the relationship between miRNAs and cancer.

52. Kent OA, Mendell JT: A small piece in the cancer puzzle: microRNAs as tumor suppressors and oncogenes. Oncogene 2006, 25:6188-6196.

53. O'Donnell WT, Warren ST: A decade of molecular studies of fragile X syndrome. Annu Rev Neurosci 2002, 25:315-338. 
54. Bardoni B, Mandel JL: Advances in understanding of fragile $\mathrm{X}$ pathogenesis and FMRP function, and in identification of $\mathrm{X}$ linked mental retardation genes. Curr Opin Genet Dev 2002, 12:284-293.

55. Jin P, Alisch RS, Warren ST: RNA and microRNAs in fragile X mental retardation. Nat Cell Biol 2004, 6:1048-1053.

56. Jin P, Zarnescu DC, Ceman S, et al.: Biochemical and genetic interaction between the fragile $\mathrm{X}$ mental retardation protein and the microRNA pathway. Nat Neurosci 2004 , 7:113-117.

57. Chen W, Jensen LR, Gecz J, et al.: Mutation screening of brain-expressed X-chromosomal miRNA genes in 464 patients with nonsyndromic X-linked mental retardation. Eur J Hum Genet 2007, 15:375-378.

58. Zhang L, Wang T, Wright AF, et al.: A microdeletion in $\mathrm{Xp11.3}$ accounts for co-segregation of retinitis pigmentosa and mental retardation in a large kindred. Am J Med Genet A 2006, 140:349-357.

59.• Abelson JF, Kwan KY, O'Roak BJ, et al.: Sequence variants in SLITRK1 are associated with Tourette's syndrome. Science 2005, 310:317-320.

This article offers evidence for an involvement of miRNAs in neurologic disorders.

60. Johnson R, Zuccato C, Belyaev ND, et al.: A microRNAbased gene dysregulation pathway in Huntington's disease. Neurobiol Dis 2008, 29:438-445.
61. Zuccato C, Tartari M, Crotti A, et al.: Huntingtin interacts with REST/NRSF to modulate the transcription of NRSEcontrolled neuronal genes. Nat Genet 2003, 35:76-83.

62. Regier DA, Narrow WE, Rae DS, et al.: The de facto US mental and addictive disorders service system. Epidemiologic catchment area prospective 1-year prevalence rates of disorders and services. Arch Gen Psychiatry 1993, 50:85-94.

63. Perkins DO, Jeffries CD, Jarskog LF, et al.: microRNA expression in the prefrontal cortex of individuals with schizophrenia and schizoaffective disorder. Genome Biol 2007, 8:R27.

64. Krutzfeldt J, Rajewsky N, Braich R, et al.: Silencing of microRNAs in vivo with 'antagomirs'. Nature 2005, 438:685-689.

65. Krutzfeldt J, Kuwajima S, Braich R, et al.: Specificity, duplex degradation and subcellular localization of antagomirs. Nucleic Acids Res 2007, 35:2885-2892.

66. Ebert MS, Neilson JR, Sharp PA: MicroRNA sponges: competitive inhibitors of small RNAs in mammalian cells. Nat Methods 2007, 4:721-726.

67. Bumcrot D, Manoharan M, Koteliansky V, Sah DW: RNAi therapeutics: a potential new class of pharmaceutical drugs. Nat Chem Biol 2006, 2:711-719. 